\title{
OPEN
}

Received: 30 November 2018

Accepted: 23 July 2019

Published online: 02 August 2019

\section{Compartmental-modelling-based measurement of murine glomerular filtration rate using ${ }^{18} \mathrm{~F}$-fluoride} PET/CT

\begin{abstract}
Hyo Sang Lee ${ }^{1}$, Yeon-koo Kang ${ }^{2}$, Hyunjong Lee ${ }^{2}$, Jeong Hee $\mathrm{Han}^{3}$, Byung Seok Moon ${ }^{3}$, Seok-Soo Byun ${ }^{4}$, Dong-Wan Chae ${ }^{5}$, Keon Wook Kang ${ }^{6,7}$ \& Won Woo Lee $\mathbb{D}^{3,8}$
\end{abstract}

Accurate measurement of glomerular filtration rate (GFR) is essential for optimal decision making in many clinical settings of renal failure. We aimed to show that GFR can be accurately measured using compartmental tracer kinetic analysis of ${ }^{18} \mathrm{~F}$-fluoride dynamic PET/CT. Twenty-three male Sprague-Dawley rats of three experimental groups (cyclosporine-administered $[n=8]$, unilaterally nephrectomized $[n=8]$, and control $[n=7]$ ) underwent simultaneous ${ }^{18} \mathrm{~F}$-fluoride dynamic PET/CT and

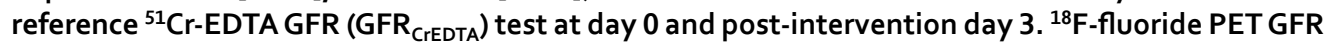
$\left(G_{F-P E T}\right)$ was calculated by multiplying the influx rate and functional kidney volume in a single-tissuecompartmental kinetic model. Within-test repeatability and between-test agreement were evaluated by intraclass correlation coefficient (ICC) and Bland-Altman analysis. In the control group, repeatability

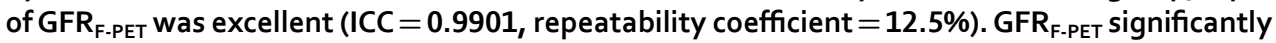
decreased in the renally impaired rats in accordance with respective $\mathrm{GFR}_{\text {CrEDTA }}$ changes. In the pooled

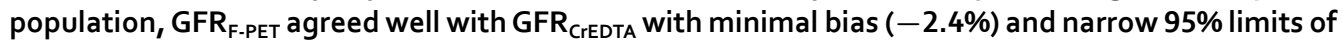
agreement (-25.0\% to $20.1 \%$ ). These data suggest that the single-compartmental kinetic analysis of ${ }^{18} \mathrm{~F}$-fluoride dynamic PET/CT is an accurate method for GFR measurement. Further studies in humans are warranted.

The glomerular filtration rate (GFR) is a widely accepted measure of global renal function, and accurate measurement of GFR is essential for optimal decision making in many clinical settings of renal failure ${ }^{1}$. The GFR has been typically measured as the urinary clearance of an ideal filtration marker such as inulin ${ }^{2}$. Alternatively, plasma clearance of a filtration marker, such as ${ }^{51} \mathrm{Cr}$-ethylenediamine-tetraacetic acid (EDTA), has been advocated for GFR measurement because of its acceptable accuracy without the necessity for tricky urine handling ${ }^{3}$. However, its drawbacks include the requirement for multiple blood samplings and a time-consuming procedure.

Nuclear medicine imaging techniques offer various means of GFR quantitation. Planar renal scintigraphy using ${ }^{99 m}$ Tc-diethylenetriamine-pentaacetic acid (DTPA) can provide imaging-based estimation of GFR via Gates' method $^{4}$. However, the GFR calculated from the Gates' formula was reported to be less accurate than measured or estimated GFR, probably due to the potential errors in the correction of background and kidney depth, inherent limitations of two-dimensional images ${ }^{5,6}$. Positron emission tomography (PET) enables dynamic 3-dimensional

${ }^{1}$ Department of Nuclear Medicine, Gangneung Asan Hospital, University of Ulsan College of Medicine, Gangneung, Republic of Korea. ${ }^{2}$ Department of Molecular Medicine and Biopharmaceutical Sciences, Graduate School of Convergence Science and Technology, Seoul National University, Seoul, Republic of Korea. ${ }^{3}$ Department of Nuclear Medicine, Seoul National University Bundang Hospital, Seoul National University College of Medicine, Seongnam-si, Republic of Korea. ${ }^{4}$ Department of Urology, Seoul National University Bundang Hospital, Seoul National University College of Medicine, Seongnam-si, Republic of Korea. ${ }^{5}$ Department of Internal Medicine, Seoul National University Bundang Hospital, Seoul National University College of Medicine, Seongnam-si, Republic of Korea. ${ }^{6}$ Department of Nuclear Medicine, Seoul National University Hospital, Seoul National University College of Medicine, Seoul, Republic of Korea. ${ }^{7}$ Cancer Research Institute, Seoul National University, Seoul, Republic of Korea. ${ }^{8}$ Institute of Radiation Medicine, Medical Research Centre, Seoul National University, Seoul, Republic of Korea. Hyo Sang Lee and Yeonkoo Kang contributed equally. Correspondence and requests for materials should be addressed to W.W.L. (email: wwlee@snu.ac.kr) 


\begin{tabular}{|l|l|l|l|l|l|l|}
\hline Group & & Number of kidneys & $\mathbf{V}_{\mathbf{C}}\left(\mathbf{c m}^{\mathbf{3}}\right)$ & $\mathbf{v B}$ & $\mathbf{K}_{\mathbf{1}}\left(\mathbf{m l} / \mathbf{c m}^{\mathbf{3}} / \mathbf{m i n}\right)$ & $\mathbf{k}_{\mathbf{2}}\left(\mathbf{m i n}^{-1}\right)$ \\
\hline \multirow{2}{*}{ Cyclosporine } & Baseline & 16 & $1.022 \pm 0.074$ & $0.111 \pm 0.034$ & $1.109 \pm 0.259$ & $0.802 \pm 0.179$ \\
\cline { 2 - 7 } & Post & 16 & $1.021 \pm 0.074$ & $0.125 \pm 0.036$ & $0.978 \pm 0.244$ & $0.764 \pm 0.215$ \\
\hline \multirow{2}{*}{ Nephrectomy } & Baseline & 16 & $1.150 \pm 0.072$ & $0.106 \pm 0.027$ & $0.967 \pm 0.175$ & $0.783 \pm 0.221$ \\
\cline { 2 - 7 } & Post & 8 & $1.178 \pm 0.057$ & $0.133 \pm 0.039$ & $1.043 \pm 0.100$ & $0.958 \pm 0.024$ \\
\hline \multirow{2}{*}{ Control } & 1 st & 14 & $1.067 \pm 0.135$ & $0.093 \pm 0.026$ & $1.009 \pm 0.269$ & $0.787 \pm 0.215$ \\
\cline { 2 - 7 } & 2nd & 14 & $1.065 \pm 0.135$ & $0.105 \pm 0.029$ & $1.043 \pm 0.277$ & $0.820 \pm 0.215$ \\
\hline
\end{tabular}

Table 1. Model parameters. The figures are expressed as mean \pm standard deviation. $\mathrm{V}_{\mathrm{C}}$, renal cortical volume; $\mathrm{vB}$, blood volume fraction; $\mathrm{K}_{1}$, influx constant; $\mathrm{k}_{2}$, efflux constant.

A

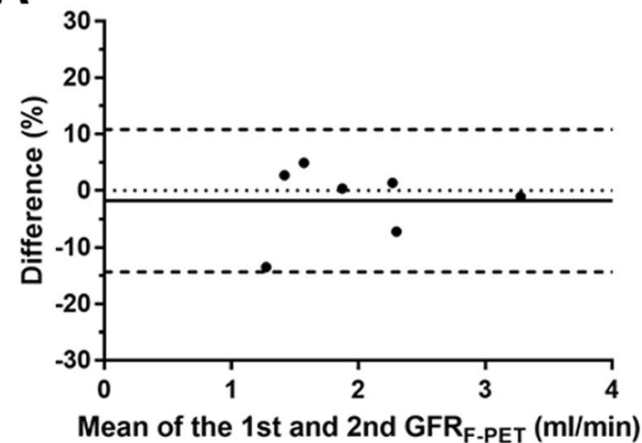

B

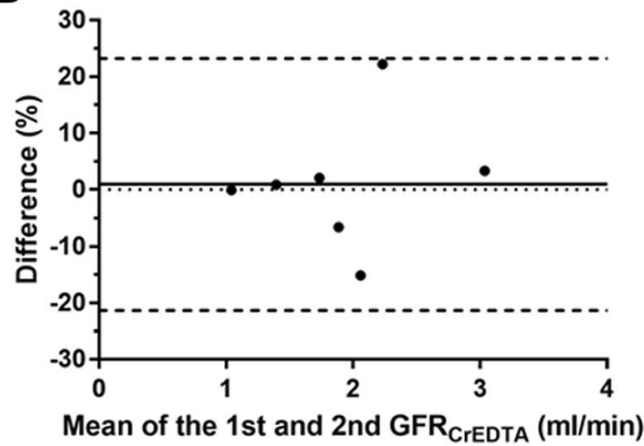

Figure 1. Bland-Altman plots for repeatability of (A) GFR $\mathrm{GFET}_{\mathrm{F}}$ and (B) $\mathrm{GFR}_{\mathrm{CrEDTA}}$. The solid lines represent biases, and the dashed lines represent $95 \%$ limits of agreement. Difference $(\%)=100 \times\left(\mathrm{GFR}_{1 \mathrm{st}}-\mathrm{GFR}_{2 \mathrm{nd}}\right) /$ (mean of $\mathrm{GFR}_{1 \mathrm{st}}$ and $\mathrm{GFR}_{2 \mathrm{nd}}$ ).

imaging, allowing accurate measurement of input function and tissue concentration of radiotracers, therefore has the potential for quantitative renal imaging ${ }^{7}$. Several proof-of-concept studies produced promising results. ${ }^{68} \mathrm{Ga}$-1,4,7-triaza-cyclononane-1,4,7-triacetic acid ( ${ }^{68} \mathrm{Ga}$-NOTA) or ${ }^{68} \mathrm{Ga}$-EDTA have been investigated for GFR measurement but the results are yet to be validated ${ }^{8,9}$. To date, there is no accepted methodological standard of PET for GFR measurement.

${ }^{18} \mathrm{~F}$-fluoride is an established skeletal PET radiopharmaceutical, but it could also be used for renal imaging because fluoride is not bound to plasma protein and thus is freely filtered through glomeruli ${ }^{10}$. However, fluoride clearance is always lower than GFR due to significant tubular reabsorption ${ }^{11,12}$. Therefore, the previous ${ }^{18} \mathrm{~F}$-fluoride dynamic PET/CT study reported a moderate correlation of fluoride clearance with a broad range of renal function parameters; the direct measurement of GFR was beyond the scope ${ }^{13}$.

Compartmental tracer kinetic modelling enables the measurement of rate constants as parameters of important physiological processes in vivo. Dynamic PET is suited for this purpose due to its accurate and non-invasive quantification ability. We hypothesized that because the compartmental modelling allows the separate quantification of influx and efflux rates, we might be able to quantify GFR using ${ }^{18} \mathrm{~F}$-fluoride influx rate despite the presence of tubular reabsorption. In this study, we showed that GFR could be accurately measured in rats via compartmental modelling of dynamic ${ }^{18} \mathrm{~F}$-fluoride PET/CT. Neither urine handling nor blood sampling was necessary in this imaging-based approach. Validity of the compartmental model was independently tested by calculating GFR using dynamic PET/CT scans of ${ }^{68} \mathrm{Ga}-\mathrm{NOTA}$.

\section{Results}

Within-test repeatability. The single-tissue-compartmental model provided excellent goodnessof-fit to the ${ }^{18} \mathrm{~F}$-fluoride renal cortical time-activity curve (TAC) (median $\mathrm{R}^{2}=0.9674$ [inter-quartile range $(I Q R)=0.9538-0.9763])$. The results of the parameter estimation are summarized in Table 1 . The renal cortical volume $\mathrm{V}_{\mathrm{C}}$ between paired measurements was highly concordant (intraclass correlation coefficient $[\mathrm{ICC}]=0.9846[95 \%$ confidence interval $(\mathrm{CI})=0.9802-0.9946]$, repeatability coefficient $=3.1 \%$ ), which suggests the reproducibility of the manual drawing of the volumes of interest (VOIs).

The repeatability of ${ }^{18} \mathrm{~F}$-fluoride PET GFR $\left(\mathrm{GFR}_{\mathrm{F}-\mathrm{PET}}\right)$ was excellent $(\mathrm{ICC}=0.9901$ [95\% CI $=0.9501-0.9982]$, repeatability coefficient $=12.5 \%)$, whereas the repeatability of ${ }^{51} \mathrm{Cr}$-EDTA GFR $\left(\mathrm{GFR}_{\mathrm{CrEDTA}}\right)$ was slightly lower than that of $\mathrm{GFR}_{\mathrm{F}-\mathrm{PET}}(\mathrm{ICC}=0.9372$ [95\% CI $=0.7155-0.9887]$, repeatability coefficient $=22.2 \%$; Fig. 1$)$.

Between-test agreement. $\quad$ GFR $_{\text {F-PET }}$ and GFR CrEDTA $_{\text {(Table 2) fell near the reported range of }{ }^{51} \mathrm{Cr}-\mathrm{EDTA}}$ plasma clearance in rats $(1.50-3.0 \mathrm{~mL} / \mathrm{min})^{14}$. Body surface areas (BSAs) of the rats were estimated as $413 \pm 16 \mathrm{~cm}^{2}\left(\right.$ range $\left.=380-455 \mathrm{~cm}^{2}\right)$. The BSA-normalized $\mathrm{GFR}_{\mathrm{F}-\mathrm{PET}}\left(\right.$ range $\left.=41.2-140.2 \mathrm{~mL} / \mathrm{min} / 1.73 \mathrm{~m}^{2}\right)$ and $\mathrm{GFR}_{\mathrm{CrEDTA}}\left(\right.$ range $\left.=44.2-127.6 \mathrm{~mL} / \mathrm{min} / 1.73 \mathrm{~m}^{2}\right)$ were well-matched with BSA-normalized human GFR. 


\begin{tabular}{|l|l|l|l|l|l|l|}
\hline \multirow{2}{*}{ Subgroup } & \multicolumn{4}{|l|}{${ }^{18}$ F-fluoride PET GFR $(\mathrm{ml} / \mathbf{m i n})$} & \multicolumn{3}{l|}{ [1 Cr-EDTA GFR $(\mathrm{ml} / \mathbf{m i n})$} \\
\cline { 2 - 7 } & Baseline & Post & $\boldsymbol{P}$ & Baseline & Post & $\boldsymbol{P}$ \\
\hline Cyclosporine & $2.01 \pm 0.43$ & $1.73 \pm 0.33$ & 0.0113 & $2.08 \pm 0.35$ & $1.82 \pm 0.38$ & 0.0300 \\
\hline Nephrectomy & $1.98 \pm 0.34$ & $1.06 \pm 0.08$ & 0.0001 & $1.97 \pm 0.35$ & $1.21 \pm 0.07$ & 0.0009 \\
\hline Control & $1.98 \pm 0.69$ & $2.01 \pm 0.69$ & 0.4415 & $1.93 \pm 0.68$ & $1.90 \pm 0.62$ & 0.7603 \\
\hline
\end{tabular}

Table 2. GFR in subgroups.

\begin{tabular}{|c|c|c|c|c|c|c|c|c|}
\hline \multirow[b]{2}{*}{ Group } & \multirow[b]{2}{*}{ ICC } & \multirow[b]{2}{*}{$95 \% \mathrm{CI}$ for ICC } & \multicolumn{2}{|c|}{ Relative difference (\%) } & \multicolumn{2}{|c|}{$\begin{array}{l}\text { Absolute difference } \\
(\mathrm{ml} / \mathrm{min})\end{array}$} & \multirow[b]{2}{*}{$\mathbf{P}_{30}$} & \multirow[b]{2}{*}{$P_{10}$} \\
\hline & & & Bias & LOA & Bias & LOA & & \\
\hline Total & 0.937 & $0.889-0.965$ & -2.4 & -25.0 to 20.1 & -0.027 & -0.401 to 0.347 & $97.8(45 / 46)$ & $60.9(28 / 46)$ \\
\hline \multicolumn{9}{|c|}{ Subgroup } \\
\hline Cys & 0.898 & $0.740-0.963$ & -4.5 & -22.9 to 13.8 & -0.080 & -0.396 to 0.236 & $100(16 / 16)$ & $81.3(13 / 16)$ \\
\hline $\mathrm{Nx}$ & 0.939 & $0.839-0.978$ & -6.5 & -28.5 to 15.5 & -0.073 & -0.395 to 0.248 & $93.8(15 / 16)$ & $50.0(8 / 16)$ \\
\hline Control & 0.941 & $0.833-0.981$ & 4.6 & -17.9 to 27.2 & 0.086 & -0.329 to 0.502 & $100(14 / 14)$ & $57.1(8 / 14)$ \\
\hline
\end{tabular}

Table 3. Accuracy statistics. ICC, intraclass correlation coefficient between ${ }^{18} \mathrm{~F}$-fluoride PET GFR and

${ }^{51}$ Cr-EDTA GFR; CI, confidence interval; LOA, limits of agreement; Cys, cyclosporine; Nx, nephrectomy.

A

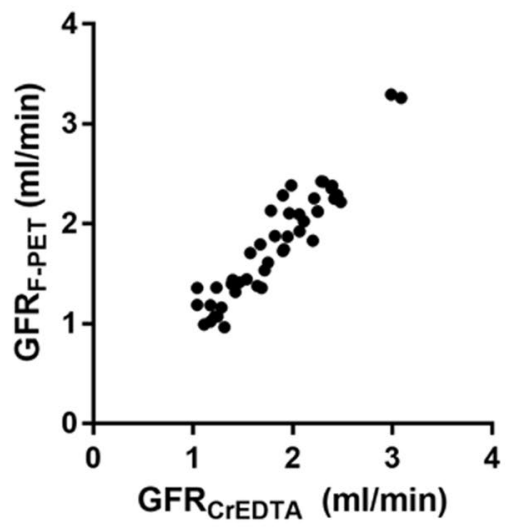

B

Total

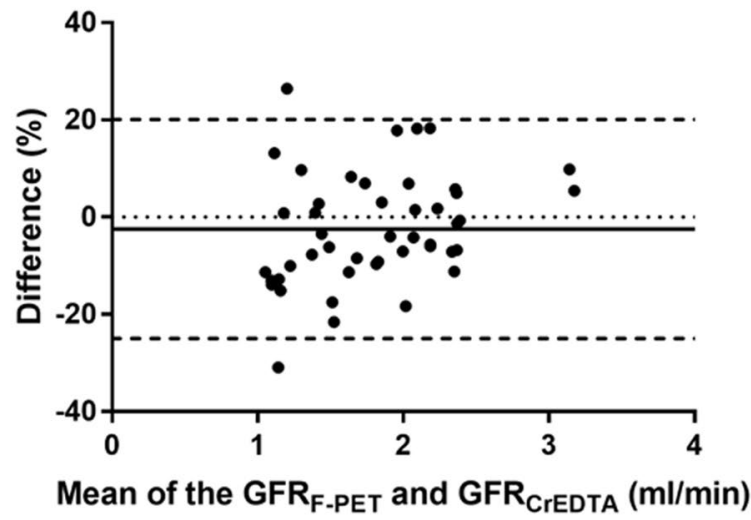

Figure 2. Agreement between $\mathrm{GFR}_{\mathrm{F}-\mathrm{PET}}$ and $\mathrm{GFR}_{\mathrm{CrEDTA}}$ in the total population (46 measurements). (A) The scatterplot. (B) The Bland-Altman plot. Difference $(\%)=100 \times\left(\mathrm{GFR}_{\mathrm{F}-\mathrm{PET}}-\mathrm{GFR}_{\mathrm{CrEDTA}}\right) /\left(\right.$ mean of GFR $\mathrm{F}_{\mathrm{PET}}$ and GFR $_{\text {CrEDTA }}$ ).

The baseline $\mathrm{GFR}_{\mathrm{F}-\mathrm{PET}}$ and $\mathrm{GFR}_{\mathrm{CrEDTA}}$ were not significantly different among the experimental groups $(P=0.830$ and 0.686 , respectively; Table 2$)$. After cyclosporine intake or nephrectomy, $\mathrm{GFR}_{\mathrm{F}-\mathrm{PET}}$ and $\mathrm{GFR}_{\mathrm{CrEDTA}}$ were significantly decreased (Supplementary Fig. 1), whereas in the control group, there was no such decrease (Supplementary Fig. 2). In each of the three groups, GFR $\mathrm{F}_{\mathrm{PET}}$ and $\mathrm{GFR}_{\mathrm{CrEDTA}}$ were in good agreement (Supplementary Fig. 3). In the pooled population (46 measurements), GFR $\mathrm{F}_{\text {-PET }}$ agreed well with GFR $_{\text {CrEDTA }}$ $(\mathrm{ICC}=0.937$ [ $95 \% \mathrm{CI}=0.889-0.965])$, with minimal bias $(-2.4 \%$ [relative difference]; $-0.027 \mathrm{ml} / \mathrm{min}$ [absolute difference]) and narrow $95 \%$ limits of agreement (LOA) ( $-25.0 \%$ to $20.1 \%$ [relative difference]; -0.401 to $0.347 \mathrm{ml} / \mathrm{min}$ [absolute difference]) (Fig. 2, Supplementary Fig. 4). $\mathrm{P}_{30}$ and $\mathrm{P}_{10}$ (see Statistics in the Methods section) were $97.8 \%(45 / 46)$ and $60.9 \%(28 / 46)$, respectively. The accuracy statistics of the GFR F-PET $_{\text {were sum- }}$ marized in the Table 3.

$\mathrm{GFR}_{\mathrm{F}-\mathrm{PET}-15 \mathrm{~min}}$ showed almost perfect agreement with $\mathrm{GFR}_{\mathrm{F}-\mathrm{PET}}(\mathrm{ICC}=0.998$ [95\% CI $=0.997-0.999]$, bias $=0.1 \%$, and $95 \% \mathrm{LOA}=-3.3 \%$ to $3.5 \%$; Supplementary Fig. 5), which suggests that the two could be used interchangeably and therefore that imaging time could be shortened to $15 \mathrm{~min}$ without loss of accuracy.

Dynamic ${ }^{68} \mathrm{Ga}-\mathrm{NOTA}$ PET/CT. Overall, ${ }^{68} \mathrm{Ga}$-NOTA showed poorer goodness-of-fit (median $\mathrm{R}^{2}=0.5223$ $[\mathrm{IQR}=0.2295-0.6528]$ for the 20 kidneys) than $\mathrm{did}{ }^{18} \mathrm{~F}$-fluoride. The discrepancy between the model curve and kidney TAC was particularly large at later time points (>about $15-20 \mathrm{~min}$ ). The goodness-of-fit was improved when only the first $15 \mathrm{~min}$ of data was used for fitting (median $\mathrm{R}^{2}=0.8557$ [IQR $\left.\left.=0.8238-0.9001\right]\right)$. Thus, we used ${ }^{68} \mathrm{Ga}-\mathrm{NOTA}$ PET GFR using first $15 \mathrm{~min}$ of data $\left(\mathrm{GFR}_{\text {NOTA-PET-15min }}\right)$ for the subsequent analysis. 
Because ${ }^{68} \mathrm{Ga}$-NOTA GFR calculation using whole-blood input function produced significant bias, conversion to plasma input function was essential (Supplementary Fig. 6A). After conversion using measured haematocrit, $\mathrm{GFR}_{\text {NOTA-PET-15min }}$ showed a good agreement with $\mathrm{GFR}_{\text {CrEDTA }}(\mathrm{ICC}=0.9664[95 \% \mathrm{CI}=0.8787-0.9914])$ with minimal bias $(-2.4 \%)$ and narrow $95 \%$ LOA ( $-25.9 \%$ to $21.1 \%$; Supplementary Fig. $6 \mathrm{~B})$. GFR NOTA-PET-15min $_{\text {using a }}$ fixed haematocrit of 0.45 showed far wider LOA $(-46.8 \%$ to $55.5 \%)$ than those using measured haematocrit (Supplementary Fig. 6C).

\section{Discussion}

In this study, we developed a compartmental tracer kinetic model for PET-based GFR measurement and applied it to ${ }^{18} \mathrm{~F}$-fluoride, which is not a GFR tracer under the conventional concept of urinary or plasma clearance measurement. According to the model, the influx rate $\mathrm{K}_{1}$ can be considered as GFR per unit extravascular renal cortical volume for any tracer that is freely filtered through glomeruli but does not undergo tubular secretion. Previous reports suggests that ${ }^{18} \mathrm{~F}$-fluoride has such properties ${ }^{11,12}$. GFR $\mathrm{F}_{\mathrm{FET}}$ was in good agreement with gold-standard GFR $_{\text {CrEDTA }}$ in conditions of nephrotoxic drug use and post-nephrectomy with minimal bias and narrow LOA. $\mathrm{P}_{30}$ and $\mathrm{P}_{10}$ were $97.8 \%$ and $60.9 \%$, respectively, which suggests that GFR $_{\text {F-PET }}$ possesses sufficient accuracy $\left(\mathrm{P}_{30}>80 \%\right.$ and $\left.\mathrm{P}_{10}>50 \%\right)$ compared with other GFR markers such as iohexol, iothalamate and DTPA ${ }^{15,16}$. Furthermore, the accuracy of $\mathrm{GFR}_{\mathrm{F}-\mathrm{PET}}$ was preserved with a reduction in imaging time to $15 \mathrm{~min}$, which bears practical importance.

Good within-test repeatability is a prerequisite for assessing between-test agreement ${ }^{17}$. The repeatability of GFR $_{\text {F-PET }}$ was excellent with repeatability coefficient (half-width of the LOA) of $12.6 \%$. GFR CrEDTA $_{\text {measured in }}$ this study showed slightly poorer repeatability coefficient of $22.2 \%$, which is somewhat large compared to the reproducibility figures previously reported in humans $(7.4-9.0 \%)^{18}$. This might have been caused by technical difficulties of the small animal experiment. We speculate that the agreement between $G_{F R} R_{F-P E T}$ and GFR CrEDTA $_{\text {. }}$ might be even better in humans, considering the expected increase in the precision of GFR $\mathrm{CrEDTA}_{\text {. }}$.

To our knowledge, approaches of measuring GFR by using a compartmental rate constant have not been attempted in the field of nuclear medicine. In contrast, various types of compartmental modelling approach have been employed in magnetic resonance imaging (MRI) or CT studies. However, a critical literature review suggested that these MRI- or CT-based methodologies are not adequately accurate to be used as routine clinical or research tools ${ }^{19}$. Among the MRI-based methods, the cortical compartment model proposed by Annet $e$ al. is similar to ours ${ }^{20}$. The differences are that Annet's method used two-dimensional regions of interest (ROIs) and abdominal aortic input function and that the dispersion and time delay from aorta to renal vasculature were accounted for. Many MRI-based methods use two-dimensional single-slice ROIs for better temporal resolution, and this acts as a limitation because a single slice or a slab cannot be representative of a whole kid$n e y^{20-22}$. In this respect, the inherent 3-dimensional capability of PET is an advantage. The use of dispersion- and time-delay-corrected aortic input curves might be a merit of Annet's method in their rabbit experiment. However, we do not think that the non-correction for dispersion and time-delay caused any significant biases in our rat experiments because of smaller animal size. If this PET/CT analysis is implemented in humans, a proper selection of site for arterial input function measurement may become an important issue.

There may be a concern about the spill-out from the renal pelvic radioactivity into the renal cortical ROIs, considering small size of the rat kidneys. However, the scatter from the renal pelvic radioactivity turned out to be negligible compared with the renal cortical uptake. No significant amount of spill-out activity from the renal pelvis reached the renal cortical ROIs because the renal cortex and renal pelvis are intervened by the renal medulla and because the spatial resolution in terms of full-width half-maximum of the micro PET system used in our study was $0.7 \mathrm{~mm}$ that was much smaller than the thickness of the renal medulla (more than $3 \mathrm{~mm}$ ).

We conducted another set of experiments using ${ }^{68} \mathrm{Ga}$-NOTA. The results also showed good agreement with GFR $_{\text {CrEDTA }}$ (Supplementary Fig. 6B). However, the goodness-of-fit to the ${ }^{68}$ Ga-NOTA data was not as good as that for ${ }^{18} \mathrm{~F}$-fluoride. The cause of the poor fit is unclear. We speculate that the urination process might not follow first-order (exponential) kinetics and therefore that the process might not be appropriately described by an exponential rate constant $\mathrm{k}_{\mathrm{u}}$. For ${ }^{68} \mathrm{Ga}-\mathrm{NOTA}$, the rate constant $\mathrm{k}_{2}\left(=\mathrm{k}_{\mathrm{u}}+\mathrm{k}_{\text {reabs }}\right)$ becomes $\mathrm{k}_{\mathrm{u}}$ because $\mathrm{k}_{\text {reabs }}=0$, and according to the above speculation, $\mathrm{k}_{2}$ also becomes an inappropriately modelled parameter. This could hamper the validity of the model equations. In contrast, ${ }^{18} \mathrm{~F}$-fluoride is reabsorbed through the lipid bilayer of tubular cells via passive diffusion ${ }^{23}$, and passive diffusion follows first-order kinetics. The reabsorption of fluoride is approximately $60 \%$ of glomerular filtrate, but it could increase up to $90 \%{ }^{11,12}$. This implies that $\mathrm{k}_{\text {reabs }}$ comprises a major portion of the efflux constant $\mathrm{k}_{2}$, causing the efflux process to roughly follow first-order kinetics. Therefore, the model fit becomes better for ${ }^{18} \mathrm{~F}$-fluoride, which would be a paradoxical advantage of nonzero reabsorption.

Measurement of haematocrit was essential for the calculation of ${ }^{68} \mathrm{Ga}$-NOTA plasma input function because the fixed plasma fraction produced imprecise GFR (Supplementary Fig. 6C). In contrast, a fixed plasma fraction of 1.23 produced accurate GFR for ${ }^{18} \mathrm{~F}$-fluoride. It is likely that the plasma fraction of ${ }^{18} \mathrm{~F}$-fluoride remained relatively stable irrespective of haematocrit because ${ }^{18} \mathrm{~F}$-fluoride permeates into the $\mathrm{RBC}^{24}$, whereas the plasma fraction of ${ }^{68} \mathrm{Ga}$-NOTA is more affected by haematocrit because ${ }^{68} \mathrm{Ga}-\mathrm{NOTA}$ cannot enter in the $\mathrm{RBC}^{8}$. The high accuracy of $\mathrm{GFR}_{\mathrm{F} \text {-PET }}$ under a fixed plasma fraction is an advantage because haematocrit need not be measured, eliminating the need for blood sampling.

Given the high accuracy of the GFR measurement using dynamic ${ }^{18} \mathrm{~F}$-fluoride PET, translational application to humans may be promising for appropriate indications. Using the expensive PET technology for GFR measurement could only be justified in clinical situations where accurate measurement of GFR is critically necessary. Such situations might include nephron-sparing surgery for malignant lesions in patients with marginal renal function, determination of overall and split renal function before abdominal radiotherapy, and monitoring of renal function during nephrotoxic drug use $\mathrm{e}^{9,25}$. 
A

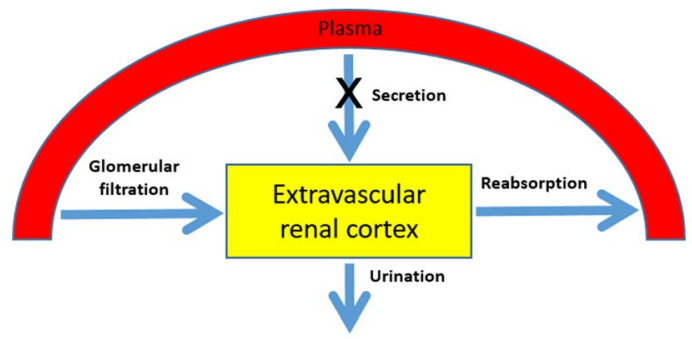

Collecting system

B

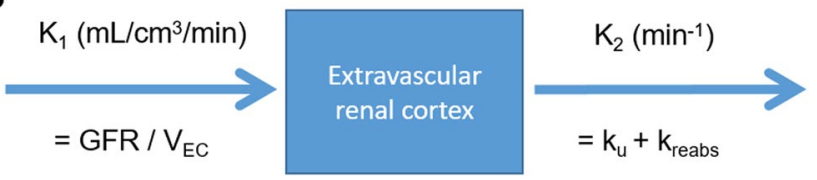

Figure 3. Study concept. (A) A schematic diagram of the single-tissue-compartmental model. (B) Rate constants in the model.

The present study has limitations. First, the range of the measured GFR was not sufficiently wide. The normalized GFR F-PET measured in this study fell within $41.2-140.2 \mathrm{~mL} / \mathrm{min} / 1.73 \mathrm{~m}^{2}$ BSA. Further validation is needed for low GFR values because chronic kidney disease stage grades 4 and 5 (GFR $<30 \mathrm{~mL} / \mathrm{min} / 1.73 \mathrm{~m}^{2}$ ) were not included in the tested range ${ }^{26}$. Second, manual drawing of ROIs is too laborious for future clinical application. Automatic segmentation of renal cortex might have to be implemented.

In conclusion, dynamic ${ }^{18} \mathrm{~F}$-fluoride PET/CT in conjunction with a single-compartmental modelling approach holds promise as a reliable and accurate method for GFR measurement. The difficulties in urine handling and blood sampling in the measurement of conventional urinary and plasma clearance of ideal filtration markers may be overcome by pure image-based analysis. A quick assessment of GFR (within $15 \mathrm{~min}$ ) is another practical advantage of this approach. Further studies in humans are warranted.

\section{Materials and Methods}

Tracer kinetic modelling. The compartmental tracer kinetic modelling is a mathematical framework that originated from the field of pharmacokinetics and is a commonly used model for analysing PET data ${ }^{27}$. In the modelling, it is assumed that there are physiologically separate pools, or compartments, of a tracer substance ${ }^{27}$. Each compartment has its own influx and efflux rate constants, and the model fitting procedure allows to quantify them. We devised a compartmental tracer kinetic model in which the rate constant of a certain compartment could be interpreted as GFR.

In the model, extravascular renal cortex (EVRC), which contains Bowman's capsule, the renal tubule, and the interstitium, serves as a functional kidney volume. A tracer enters the EVRC via glomerular filtration and tubular secretion and moves out via reabsorption and urinary outflow (Fig. 3A). The rate of change in the tracer amount within the EVRC can be described by the following equation:

$$
\begin{aligned}
\frac{d A_{E C}(t)}{d t} & =G F R \times C_{P}(t)+k_{\text {secr }} \times C_{P}(t)-k_{u} \times A_{E C}(t)-k_{\text {reabs }} \times A_{E C}(t) \\
& =\left(G F R+k_{\text {secr }}\right) \times C_{P}(t)-\left(k_{u}+k_{\text {reabs }}\right) \times A_{E C}(t)
\end{aligned}
$$

where $A_{E C}(t)=$ tracer amount within EVRC, $C_{P}(t)=$ tracer concentration in plasma, $\mathrm{k}_{\text {secr }}=$ rate constant of tubular secretion, $\mathrm{k}_{\mathrm{u}}=$ rate constant of tracer loss due to urinary outflow from the cortex, and $\mathrm{k}_{\text {reabs }}=$ rate constant of tubular reabsorption.

Because no tubular secretion occurs for the ${ }^{18} \mathrm{~F}-$ fluoride $^{11,12}, \mathrm{k}_{\text {secr }}=0$ (Fig. 3A), the Equation (1) becomes as follows:

$$
\frac{d A_{E C}(t)}{d t}=G F R \times C_{P}(t)-\left(k_{u}+k_{r e a b s}\right) \times A_{E C}(t)
$$

Dividing the equation by EVRC volume $\mathrm{V}_{\mathrm{EC}}=\mathrm{V}_{\mathrm{C}} \times(1-\mathrm{vB})$ gives

$$
\begin{aligned}
& \frac{d}{d t}\left(A_{E C}(t) / V_{E C}\right)=\frac{G F R}{V_{E C}} \times C_{P}(t)-\left(k_{u}+k_{\text {reabs }}\right) \times \frac{A_{E C}(t)}{V_{E C}} \\
& \frac{d C_{E C}(t)}{d t}=K_{1} \times C_{P}(t)-k_{2} \times C_{E C}(t)
\end{aligned}
$$

where $\mathrm{V}_{\mathrm{C}}=$ renal cortical volume, $\mathrm{vB}=$ vascular volume fraction, $\mathrm{C}_{\mathrm{EC}}(\mathrm{t})$ = tracer concentration within the EVRC, $\mathrm{K}_{1}=\mathrm{GFR} / \mathrm{V}_{\mathrm{EC}}$ and $\mathrm{k}_{2}=\mathrm{k}_{\mathrm{u}}+\mathrm{k}_{\text {reabs }}$ (Fig. $3 \mathrm{~B}$ ).

The solution to Equation (3) can be expressed as follows: 
A

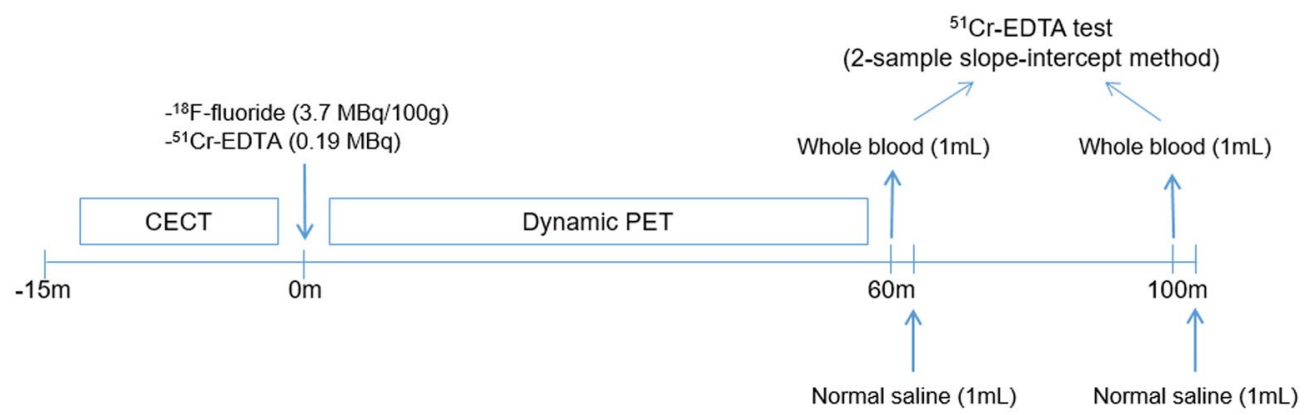

B - Medical renal impairment model

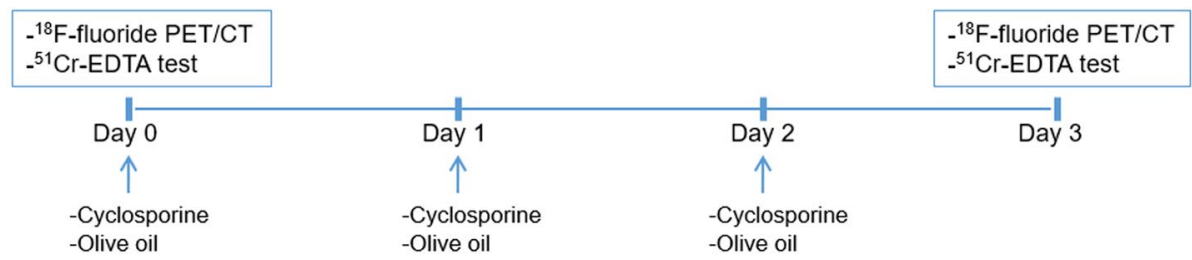

- Surgical renal impairment model

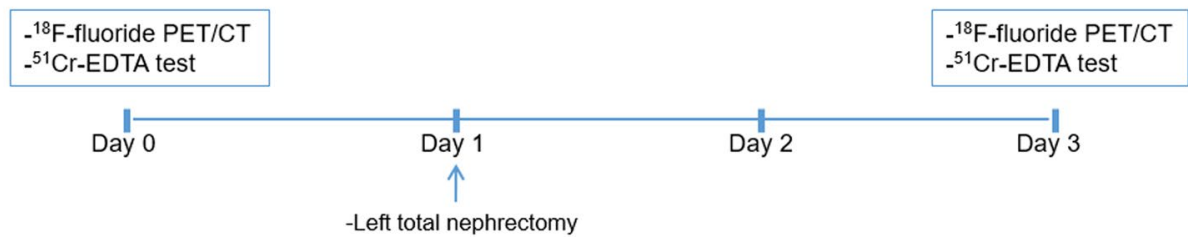

Figure 4. Study design. (A) ${ }^{18} \mathrm{~F}$-fluoride dynamic PET/CT imaging and ${ }^{51} \mathrm{Cr}$-EDTA test protocol. $\mathrm{CECT}=$ contrast-enhanced computed tomography. (B) Animal experiment protocol.

$$
C_{E C}(t)=K_{1} \times \int_{0}^{t} C_{P}(\tau) e^{-k_{2}(t-\tau)} d \tau=K_{1} \times C_{P}(t) \otimes e^{-k_{2} t}
$$

where $\otimes=$ convolution integral.

The model function $\mathrm{C}_{\text {model }}(\mathrm{t})$ can be expressed as a superposition of $\mathrm{C}_{\mathrm{EC}}(\mathrm{t})$ and $\mathrm{C}_{\mathrm{P}}(\mathrm{t})$ according to their respective volume fractions in the kidney:

$$
C_{\text {model }}(t)=C_{E C}(t) \times(1-v B)+C_{P}(t) \times v B
$$

The $\mathrm{C}_{\text {model }}(\mathrm{t})$ is fitted to the renal cortical TAC with $\mathrm{K}_{1}, \mathrm{k}_{2}$, and $\mathrm{vB}$ as fitting parameters. Single-kidney GFR is obtained by multiplying $\mathrm{K}_{1}$ and $\mathrm{V}_{\mathrm{C}} \times(1-\mathrm{vB})$, and total GFR is the sum of the GFR values of both kidneys.

We applied the above model to ${ }^{18} \mathrm{~F}$-fluoride dynamic PET/CT to measure the GFR and compared the values with gold-standard ${ }^{51} \mathrm{Cr}$-EDTA GFR. Additionally, we tested the model using ${ }^{68} \mathrm{Ga}$-NOTA. ${ }^{68} \mathrm{Ga}$-NOTA was recently reported as a promising GFR tracer with no tubular reabsorption and secretion, and minimal binding to RBCs and serum protein ${ }^{8}$.

Radiopharmaceutical preparation. ${ }^{18} \mathrm{~F}$-fluoride was produced by proton irradiation to the $\mathrm{H}_{2}{ }^{18} \mathrm{O}$ target using an in-house cyclotron (KOTRON-13, Samyoung Unitech). ${ }^{68} \mathrm{Ga}-\mathrm{NOTA}$ was produced by labelling NOTA (ChemaTech) with ${ }^{68} \mathrm{Ga}$ eluted from a ${ }^{68} \mathrm{Ge} /{ }^{68} \mathrm{Ga}$ generator (IGG100; Eckert \& Ziegler) as previously described ${ }^{8}$.

Protocol of ${ }^{18} \mathrm{~F}$-fluoride dynamic PET/CT Imaging and the ${ }^{51} \mathrm{Cr}$-EDTA Test. Imaging was performed from the thorax to the abdomen in the prone position on a dedicated small-animal PET/CT scanner (NanoScan micro PET/CT 122S; Mediso) under general anaesthesia through isoflurane inhalation $(2-3 \%$ in $2-5 \mathrm{~L} / \mathrm{min}$ of oxygen). In each PET/CT imaging sessions, ${ }^{18} \mathrm{~F}$-fluoride $(3.7 \mathrm{MBq} / 100 \mathrm{~g}$ rat weight in $200 \mu \mathrm{L}$ solution) and ${ }^{51} \mathrm{Cr}$-EDTA (GE Healthcare; $0.19 \mathrm{MBq}$ in $500 \mu \mathrm{L}$ solution) were simultaneously injected via the tail vein after the acquisition of the contrast-enhanced CT scan. Immediately following the injection of the radiopharmaceuticals, dynamic ${ }^{18} \mathrm{~F}$-fluoride PET images were obtained in the list mode for $60 \mathrm{~min}$ with varying frame durations $(5 \mathrm{~s} \times 6$, $10 s \times 3,15 s \times 4,30 s \times 16,60 s \times 20$, and $300 s \times 6)($ please see the Supplementary Methods for PET/CT parameters for acquisition and reconstruction).

After the dynamic PET acquisition, at 60 and 100 min post ${ }^{51} \mathrm{Cr}$-EDTA injection, $1 \mathrm{~mL}$ of blood was withdrawn via tail-tip cutting (Fig. 4A). Following each blood withdrawal, $1 \mathrm{~mL}$ of saline was flushed to replenish the volume. Plasma samples obtained by centrifugation $(3,000 \mathrm{rpm}$ for $8 \mathrm{~min})$ were divided into two aliquots for 

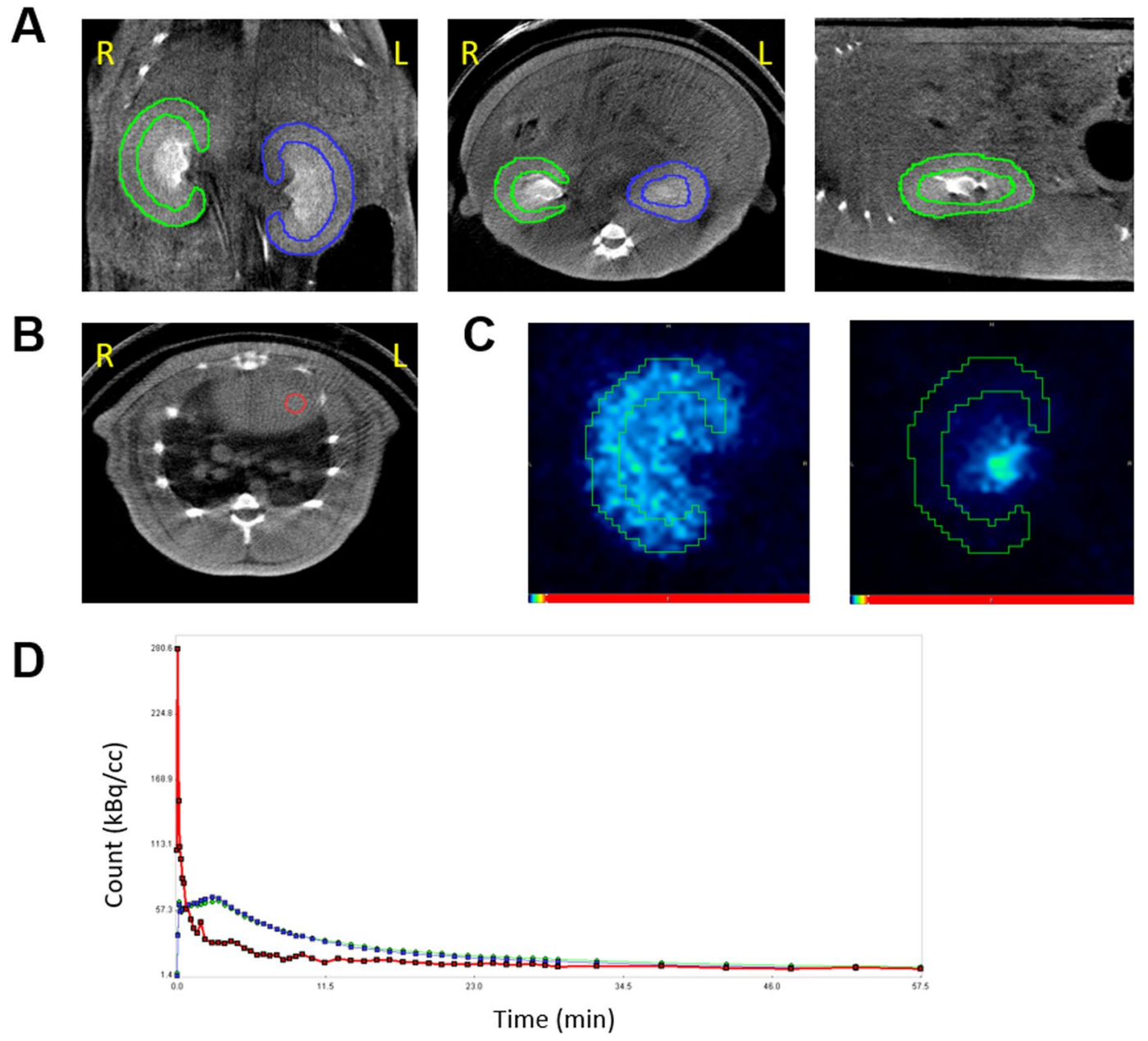

Figure 5. How to analyze the ${ }^{18} \mathrm{~F}$-fluoride dynamic PET/CT. (A) Renal cortical regions of interest. (B) The left ventricular volume of interest. (C) ${ }^{18} \mathrm{~F}$-fluoride PET images in the renal uptake phase ( 2.5 to 3 min postinjection; left panel) and excretory phase (25 to $26 \mathrm{~min}$ post-injection; right panel). (D) Time-activity curves of the right kidney (green), left kidney (blue), and left ventricle (red). $\mathrm{R}=$ right, $\mathrm{L}=$ left.

duplication, and the radioactivity of the plasma aliquots was measured for 20 min using a well counter (Wizard 1480, Perkin Elmer) $24 \mathrm{~h}$ after the blood withdrawal to ensure full decay of the PET radiopharmaceuticals. The plasma clearance of ${ }^{51} \mathrm{Cr}$-EDTA was calculated from the mean values of the duplicate counts after background correction using the two-sample slope-intercept method ${ }^{28}$. The slope-intercept plasma clearance was corrected for neglecting the fast exponential in the bi-exponential plasma curve, generating the $\mathrm{GFR}_{\mathrm{CrEDTA}}$ (please see the Supplementary Methods for details ${ }^{29}$.

Animal experiment protocol. For the ${ }^{18} \mathrm{~F}$-fluoride PET/CT experiment, 23 male Sprague-Dawley rats (age: 8 weeks; weight: $280 \pm 12 \mathrm{~g}$ ) were used. The rats were divided into three experimental groups. Eight rats were administered with cyclosporine (Sandimmun INJ, Novartis) $30 \mathrm{mg} / \mathrm{kg}$ orally from day 0 to 2 to induce renal impairment medically. Another eight rats underwent left total nephrectomy at day 1 to form a surgical renal impairment group. The remaining seven rats were fed $1 \mathrm{~mL} /$ day olive oil from day 0 to 2 and served as controls. Each rat underwent two ${ }^{18} \mathrm{~F}$-fluoride PET/CT imaging sessions at an interval of 3 days, at baseline (day 0 ) and after the renal impairment or control procedures (day 3) (Fig. 4B).

For the ${ }^{68} \mathrm{Ga}$-NOTA PET/CT experiment, 10 male naïve Sprague-Dawley rats ( $334 \pm 52 \mathrm{~g}$ ) underwent dynamic PET/CT and a ${ }^{51} \mathrm{Cr}$-EDTA test. The experimental protocol was the same for the ${ }^{68} \mathrm{Ga}$-NOTA experiment, except for the haematocrit measurement (please see Supplementary Methods) and ${ }^{68} \mathrm{Ga}-\mathrm{NOTA}(3.7 \mathrm{MBq} / 100 \mathrm{~g}$ rat weight) injection.

Image analysis. We performed PET/CT data analysis and tracer kinetic modelling using PMOD software (version 3.8; PMOD Technologies). ROIs were manually drawn over the renal cortices on the coronal CT images (Fig. 5A), and the ROIs over the same kidney were integrated to form a VOI. A 3-mm-diameter spherical VOI was placed in the left ventricular cavity to obtain whole-blood input function (Fig. 5B). The ROIs was overlaid on the co-registered dynamic PET images to obtain renal cortical TACs (Fig. 5C). In order to convert whole-blood 
input function to plasma input function, we adopted a fixed plasma fraction of 1.23 for ${ }^{18} \mathrm{~F}$-fluoride ${ }^{30}$ because it permeates into RBCs with its intracellular concentration stable with about half in plasma ${ }^{31,32}$. In contrast, we adopted a plasma fraction of $1 /(1-$ hematocrit $)$ for ${ }^{68} \mathrm{Ga}$-NOTA because it does not distribute into $\mathrm{RBCs}^{8}$. To test whether the measurement of haematocrit is mandatory for the calculation of ${ }^{68} \mathrm{Ga}$-NOTA plasma input function, we calculated another set of plasma input functions by assuming a fixed haematocrit of 0.45 .

The single-tissue-compartmental model curve using the plasma input function was fitted to the renal cortical TACs to obtain GFR F-PET $_{\text {and }}{ }^{68}$ Ga-NOTA PET GFR (GFR ${ }_{\text {NOTA-PET }}$ ) (Fig. 5D). Additionally, we calculated PET GFR only using the first $15 \mathrm{~min}$ of data $\left(\mathrm{GFR}_{\mathrm{F}-\mathrm{PET}-15 \mathrm{~min}}\right.$ and $\left.\mathrm{GFR}_{\mathrm{NOTA}-\mathrm{PET}-15 \mathrm{~min}}\right)$ to test the feasibility of reducing imaging time.

Statistics. The goodness-of-fit of the model was assessed using the coefficient of determination $\left(\mathrm{R}^{2}\right)$. We used the control group data to test for repeatability. Within-test repeatability and between-test agreement were assessed by means of the ICC and the Bland-Altman analysis ${ }^{17,33}$. Accuracy of GFR $\mathrm{F}_{\text {-PET }}$ was expressed by $\mathrm{P}_{30}$ and $\mathrm{P}_{10}$, which are defined as the percentages of the measurements that lie within the $\pm 30 \%$ and $\pm 10 \%$ ranges from reference $\mathrm{GFR}_{\mathrm{CrEDTA}}$, respectively ${ }^{15,16}$. The paired-samples $t$-test was performed to analyse the difference between paired observations. The Kruskal-Wallis test was performed for group comparisons. Two-sided $P<0.05$ was considered as significant. All statistical tests were performed using MedCalc statistical software (version 18.5; MedCalc Software bvba).

Study approval. The rats were cared for in a facility accredited by the Association for Assessment and Accreditation of Laboratory Animal Care International. The study protocol was approved by the Institutional Animal Care and Use Committee of Seoul National University Bundang Hospital (IACUC No. BA1705-223/04101). All experiments were performed in accordance with relevant guidelines and regulations.

\section{Data Availability}

The datasets generated during and/or analysed during the current study are available from the corresponding author on reasonable request.

\section{References}

1. Go, A. S., Chertow, G. M., Fan, D., McCulloch, C. E. \& Hsu, C. Chronic kidney disease and the risks of death, cardiovascular events, and hospitalization. N. Engl. J. Med., https://doi.org/10.1056/NEJMoa041031 (2004)

2. Stevens, L. A. \& Levey, A. S. Measured GFR as a Confirmatory Test for Estimated GFR. J. Am. Soc. Nephrol. 20, 2305-2313 (2009).

3. Fleming, J. S., Zivanovic, M. A., Blake, G. M., Burniston, M. \& Cosgriff, P. S. Guidelines for the measurement of glomerular filtration rate using plasma sampling. Nucl. Med. Commun. 25, 759-769 (2004).

4. Gates, G. F. Glomerular filtration rate: estimation from fractional renal accumulation of 99mTc-DTPA (stannous). AJR. Am. J. Roentgenol. 138, 565 (1982).

5. De Santo, N. G. et al. Measurement of glomerular filtration rate by the $99 \mathrm{mTc}-\mathrm{DTPA}$ renogram is less precise than measured and predicted creatinine clearance. Nephron 81, 136-140 (1999).

6. Ma, Y.-C. et al. Comparison of 99mTc-DTPA renal dynamic imaging with modified MDRD equation for glomerular filtration rate estimation in Chinese patients in different stages of chronic kidney disease. Nephrol. Dial. Transplant. 22, 417-423 (2006).

7. Szabo, Z., Xia, J., Mathews, W. B. \& Brown, P. R. Future direction of renal positron emission tomography. Seminars in Nuclear Medicine 36, 36-50 (2006).

8. Lee, J. Y. et al. Preparation of Ga-68-NOTA as a renal PET agent and feasibility tests in mice. Nucl. Med. Biol. 41, 210-215 (2014).

9. Hofman, M. et al. 68Ga-EDTA PET/CT imaging and plasma clearance for glomerular filtration rate quantification: comparison to conventional 51Cr-EDTA. J. Nucl. Med. 56, 405-9 (2015).

10. Zohoori, F. V., Innerd, A., Azevedo, L. B., Whitford, G. M. \& Maguire, A. Effect of exercise on fluoride metabolism in adult humans: A pilot study. Sci. Rep. 5, 1-9 (2015).

11. Järnberg, P. O., Ekstrand, J. \& Ehrnebo, M. Renal excretion of fluoride during water diuresis and induced urinary ph-changes in man. Toxicol. Lett., https://doi.org/10.1016/0378-4274(83)90084-X (1983).

12. Spak, C. J., Berg, U. \& Ekstrand, J. Renal clearance of fluoride in children and adolescents. Pediatrics 75, 575-9 (1985).

13. Schnöckel, U. et al. Dynamic 18F-fluoride small animal PET to noninvasively assess renal function in rats. Eur. J. Nucl. Med. Mol. Imaging 35, 2267-2274 (2008).

14. Seefeldt, T. Plasma Clearance of 51Cr-EDTA as an Estimator of Glomerular Filtration Rate in Conscious Rats. J. Appl. Toxicol. 10, 439-442 (1990)

15. Stevens, L. A., Zhang, Y. \& Schmid, C. H. Evaluating the performance of equations for estimating glomerular filtration rate. J. Nephrol. 21, 797-807 (2008).

16. Soveri, I. et al. Measuring GFR: A systematic review. Am. J. Kidney Dis. 64, 411-424 (2014).

17. Martin, B. J. \& Altman, D. Statistical Methods for Assessing Agreement Between Two Methods of Clinical Measurement. Lancet, https://doi.org/10.1016/S0140-6736(86)90837-8 (1986).

18. Delanaye, P., Cavalier, E., Froissart, M. \& Krzesinski, J. M. Reproducibility of GFR measured by chromium-51-EDTA and iohexol. Nephrol. Dial. Transplant. 23, 4077-4078 (2008).

19. Mendichovszky, I. et al. How accurate is dynamic contrast-enhanced MRI in the assessment of renal glomerular filtration rate? A critical appraisal. J. Magn. Reson. Imaging 27, 925-931 (2008).

20. Annet, L. et al. Glomerular filtration rate: Assessment with dynamic contrast-enhanced MRI and a cortical-compartment model in the rabbit kidney. J. Magn. Reson. Imaging 20, 843-849 (2004).

21. Niendorf, E. R., Grist, T. M., Lee, F. T., Brazy, P. C. \& Santyr, G. E. Rapid in vivo measurement of single-kidney extraction fraction and glomerular filtration rate with MR imaging. Radiology 206, 791-798 (1998).

22. Hackstein, N., Heckrodt, J. \& Rau, W. S. Measurement of Single-Kidney Glomerular Filtration Rate Using a Contrast-Enhanced Dynamic Gradient-Echo Sequence and the Rutland-Patlak Plot Technique. J. Magn. Reson. Imaging 18, 714-725 (2003).

23. Buzalaf, M. A. R. \& Whitford, G. M. Fluoride metabolism. In Fluoride and the Oral Environment, https://doi.org/10.1159/000325107 (2011).

24. Park-Holohan, S. J., Blake, G. M. \& Fogelman, I. Quantitative studies of bone using (18)F-fluoride and (99m)Tc-methylene diphosphonate: evaluation of renal and whole-blood kinetics. Nucl. Med. Commun. 22, 1037-44 (2001).

25. Blaufox, M. D. PET Measurement of Renal GFR: Is there a role in Nuclear Medicine. J. Nucl. Med. 1495-1497, https://doi. org/10.2967/jnumed.116.174607 (2016). 
26. KDIGO 2017 Clinical Practice Guideline Update for the Diagnosis, Evaluation, Prevention, and Treatment of Chronic Kidney Disease-Mineral and Bone Disorder (CKD-MBD). Kidney Int. Suppl. 7, 1-59 (2017).

27. Watabe, H., Ikoma, Y., Kimura, Y., Naganawa, M. \& Shidahara, M. PET kinetic analysis - Compartmental model. Ann. Nucl. Med. 20, 583-588 (2006)

28. Blaufox, M. D. et al. Report of the Radionuclides in Nephrourology Committee on Renal Clearance. J. Urol. 2297, https://doi. org/10.1097/00005392-199812010-00116 (1998).

29. Fleming, J. S. An improved equation for correcting slope-intercept measurements of glomerular filtration rate for the single exponential approximation. Nucl. Med. Commun. 28, 315-320 (2007).

30. Hawkins, Ra et al. Evaluation of the skeletal kinetics of fluorine-18-fluoride ion with PET. J. Nucl. Med. 33, 633-42 (1992).

31. Charkes, N. D., Brookes, M. \& Makler, P. T. Studies of skeletal tracer kinetics: II. evaluation of a five-compartment model of [18F] fluoride kinetics in rats. J. Nucl. Med. 20, 1150-7 (1979).

32. Schiepers, C. et al. Fluoride kinetics of the axial skeleton measured in vivo with fluorine-18-fluoride PET. J. Nucl. Med. 38, 1970-1976 (1997).

33. Shrout, P. E. \& Fleiss, J. L. Intraclass correlations: uses in assessing rater reliability.1. Shrout PE, Fleiss JL: Intraclass correlations: uses in assessing rater reliability. Psychol Bull 86, 420-8 (1979).

\section{Acknowledgements}

This work was supported in part by the research fund of Gangneung Asan Hospital (2017S002) and Seoul National University Bundang Hospital (14-2016-012).

\section{Author Contributions}

W.L. and H.S.L. designed research. Y.K. and H.J. conducted experiments. H.S.L., Y.K., H.L., H.J. and B.M. acquired data. H.S.L., Y.K. and W.L. analysed data. S.B., D.C. and K.K. gave critique. H.S.L., Y.K., H.J. and W.L. wrote the manuscript.

\section{Additional Information}

Supplementary information accompanies this paper at https:/doi.org/10.1038/s41598-019-47728-x.

Competing Interests: The authors declare no competing interests.

Publisher's note: Springer Nature remains neutral with regard to jurisdictional claims in published maps and institutional affiliations.

(c) Open Access This article is licensed under a Creative Commons Attribution 4.0 International

License, which permits use, sharing, adaptation, distribution and reproduction in any medium or format, as long as you give appropriate credit to the original author(s) and the source, provide a link to the Creative Commons license, and indicate if changes were made. The images or other third party material in this article are included in the article's Creative Commons license, unless indicated otherwise in a credit line to the material. If material is not included in the article's Creative Commons license and your intended use is not permitted by statutory regulation or exceeds the permitted use, you will need to obtain permission directly from the copyright holder. To view a copy of this license, visit http://creativecommons.org/licenses/by/4.0/.

(C) The Author(s) 2019 